\title{
¿QUÉ DEBEN ESPERAR LAS MUJERES DE UN ESTADO LAICO?
}

\section{WHAT SHOULD WOMEN EXPECT OF A SECULAR STATE?}

\author{
Isabel TURÉGANO \\ Universidad de Castilla la Mancha
}

Recibido: $12 / 6 / 2016$

Aceptado: 5/9/2016

Para citar este artículo / To cite this article:

Turégano, Isabel. «¿Qué deben esperar las mujeres de un Estado laico?». En Nieves Montesinos Sánchez y Beatriz Souto Galván (coords.), Laicidad y creencias. Feminismo/s, 28 (diciembre 2016): 49-74, DOI: 10.14198/fem.2016.28.02

Para enlazar con este artículo / To link to this article:

http://dx.doi.org/10.14198/fem.2016.28.02

\section{Resumen}

La libertad religiosa es un principio básico de cualquier sistema jurídico-político que asuma la relevancia esencial de la libertad humana. Sin embargo, otros derechos igualmente básicos pueden verse afectados si esa libertad se interpreta como autonomía de lo religioso respecto de lo político, protegiendo a los grupos religiosos como realidades dadas y cerradas. La libertad religiosa en su sentido más coherente con la igualdad de género debe requerir que el resto de derechos no sea afectado de modo discriminatorio por la actuación de las comunidades religiosas. Ello no supone una contradicción necesaria entre género y religión si se asume una concepción abierta y plural de las religiones como comunidades de conversación. Desde ésta, la libertad religiosa no es incompatible con la legítima función de las instituciones políticas, estatales e internacionales, de promover los elementos igualitarios de las tradiciones religiosas, incentivando la discusión interna y apoyando a los grupos internos que tratan de superar las discriminaciones. Unas instituciones verdaderamente comprometidas con la libertad religiosa deberían actuar porque las mujeres dejen de ser mero objeto de debate y pasen a ser agentes en el mismo, con un papel activo y reflexivo en su propia tradición religiosa. Una concepción 
diferente de las religiones como realidades holísticas y estáticas y favorable a tratar los argumentos religiosos como excepción a los modelos normativos generales es incompatible con la laicidad y la igualdad de género que ésta debe especialmente promover.

Palabras clave: Laicidad, igualdad de género, Estado, religión, cultura.

\begin{abstract}
Freedom of religion is a basic principle to any legal and political system that assumes the essential relevance of human freedom. However, other equally basic rights can be affected if that freedom is interpreted as autonomy of religion with respect to politics, protecting religious groups as given and closed realities. In its more consistent sense with gender equality, freedom of religion should require that other rights are not affected by intervention of religious communities. This does not involve a necessary contradiction between gender and religion if an open and plural conception of religions as communities of conversation is assumed. From this, religious freedom is not incompatible with the legitimate function of political institutions, domestic and international, to promote the egalitarian elements of religious traditions, encouraging internal debate and supporting the internal groups that try to overcome discrimination. Institutions truly committed to religious freedom should act so as women are no longer treated as mere subject of debate but actors in it, with a reflective and active role in their own religious tradition. A different conception of religions as holistic and static realities and favorable to treat religious arguments as exception to general normative models is incompatible with secularism and the gender equality that it should especially promote.
\end{abstract}

Keywords: Secularism, gender equality, State, religion, culture. 


\section{PLANTEAMIENTO}

El proyecto emancipador de las mujeres sigue en nuestros días viéndose postergado en aras de prioridades económicas, políticas, o identitarias que se anteponen a la consecución de la igualdad. Entre tales factores, la creciente influencia de las religiones en nuestras sociedades contribuye a obstaculizar la igualación social de las mujeres. Esta afirmación no presupone que la religión, cualquier religión, tenga necesariamente un carácter discriminatorio en cuanto tal. Realmente, los elementos discriminatorios que conllevan las religiones tienen su base fundamentalmente en estructuras sociales y culturales que se entrelazan con ellas. Pero las religiones, tanto por el dominio ideológico que pueden ejercer como por su grado de desarrollo institucional, son un recurso importante de poder y movilización que refuerzan esa discriminación. Su especial capacidad para ello deriva de su pretensión de poseer la verdad y ser capaces de conformar los compromisos éticos más básicos de los sujetos, dotando a quienes conocen y transmiten esas verdades de autoridad moral incuestionable. Como tal recurso, en la medida en que contienen una doctrina formulada casi exclusivamente por varones e históricamente han interiorizado un modelo de mujer sumisa y pasiva, las religiones contribuyen a reforzar y legitimar la discriminación sistémica en nuestras sociedades. No obstante, el modo en que las religiones han tratado tradicionalmente la igualdad de género no excluye la posibilidad de tratamientos diversos, en la medida en que las religiones, que son realidades heterogéneas que contienen voces plurales, sean capaces de incorporar planteamientos alternativos de sus principios y valoraciones (Nussbaum 1999, 115).

El presente trabajo plantea, en primer lugar, los aspectos que hacen que un Estado laico sea el más idóneo para avanzar hacia la progresiva eliminación de la desigualdad de género. Así es, en primer lugar, si se asume un concepto neutral de laicidad que no se limite a su dimensión pasiva sino también activa a favor de la igual libertad de todos en la elección de sus ideas y creencias; y si se entiende, en segundo lugar, que un modelo laico debe superar el hecho de que la participación de las mujeres en lo público se vea mediada por posiciones religiosas. La deseable ampliación de la deliberación a las posiciones 
plurales de los sujetos no puede hacerse sin algunas restricciones relevantes relativas a la proscripción del dogmatismo y la posibilidad de amplias esferas de discrecionalidad individual.

Pero las religiones se consideran especialmente merecedoras de un peso particular en la esfera pública que les dotaría de la capacidad de determinar posibles restricciones de derechos colectivamente reconocidos. La segunda parte del trabajo trata de mostrar la insuficiencia de los argumentos que se aducen para fundar esa tesis y la dificultad de trazar una línea divisoria entre prácticas religiosas y culturales. Esta perspectiva permite plantear la necesidad de un concepto abierto y dinámico de las religiones que admite la disidencia en su seno. Ello supone proponer un concepto de libertad religiosa compatible con la legítima función de las instituciones públicas de promover la participación de las mujeres para reducir el desequilibrio de poder y favorecer una resignificación del legado de principios y prácticas. El trabajo termina planteando tres instrumentos de actuación pública que deniegan esa concepción de la libertad religiosa coherente con la igualdad de género y ocultan el debate interno que condiciona el peso de los argumentos religiosos: las excepciones a leyes generales, la denominada «defensa cultural» en Derecho penal y las reservas a las documentos jurídicos internacionales de protección de los derechos de las mujeres. En los tres casos, la pretensión de garantía de la identidad religiosa refuerza la subordinación sistémica de las mujeres.

\section{LAICIDAD, AUTONOMÍA Y DEMOCRACIA}

Profundizar en un modelo estatal laico es necesario para avanzar hacia la superación de la subordinación estructural de las mujeres. La laicidad tiene que ver con tres aspectos de un modelo legítimo de Estado que afectan especialmente a la igualdad de género: en primer lugar, aun entendida en un sentido neutral, la laicidad no es posible sin una preocupación del Estado por la efectiva libertad e igualdad de todos. Ello implica un sentido de «neutralidad activa» favorable a la actuación pública en favor de la igualdad de los miembros de los grupos religiosos. En segundo lugar, la laicidad supone la necesidad de un debate público plural y racional que enfrente la pretensión de las confesiones religiosas de imponer su percepción de la moral como única correcta. Y, por último, la laicidad debe abrirse, más allá de una mera actitud del Estado ante nuestras convicciones más profundas, hacia la igual capacidad efectiva de realizar los derechos básicos. 


\subsection{Neutralidad activa y derechos}

En primer lugar, en un modelo estatal laico, la libertad religiosa ha de demandar de los poderes públicos imparcialidad respecto del fenómeno religioso. En su versión más militante o radical, ello implica la prohibición de toda manifestación externa de cultos religiosos, yendo más allá de una razonable exclusión de la religión del ámbito político. En una versión positiva o abierta de la laicidad se asume que la declaración constitucional de no confesionalidad admite un cierto compromiso entre el Estado y las Iglesias y una neutralidad limitada que evita la interferencia coactiva en las creencias religiosas pero sin que el Estado se abstenga de favorecer ciertas posiciones religiosas. Esta forma de laicidad, a la que puede asimilarse nuestro modelo constitucional, asume una forma de confesionalidad genérica o formalmente universal que favorece medidas positivas y prestacionales respecto de las religiones (aunque en la práctica no respecto de todas en igual medida). Entre ambas versiones, Ruiz Miguel propone un modelo de «laicidad neutral» para el que el Estado debe ser imparcial no solo entre las diferentes religiones sino también, en general, en materia religiosa (Ruiz Miguel 2013). En este modelo, es función del Estado proteger la libertad religiosa, sin que ello suponga un exceso de protección de algunas posiciones religiosas en detrimento de una libertad plena que ampare también la expresión de ideas no religiosas. Esta versión de la laicidad, entendida como estricta neutralidad ante toda convicción en materia de religión, no tiene por qué implicar necesariamente un comportamiento pasivo de las instituciones públicas. Que estas deban ser neutrales respecto de las convicciones éticas no es incompatible con su deber de favorecer que los grupos e instituciones religiosas hagan posible la efectiva libertad religiosa de todo individuo. Es esta versión activa de la neutralidad la que resulta más adecuada para la superación de la discriminación de las mujeres.

La neutralidad supone un principio de legitimidad estatal independiente de la realidad de creencias más o menos mayoritarias en cada contexto político. La neutralidad en su vertiente pasiva debe entenderse en el sentido de igual respeto hacia las personas de diferentes grupos religiosos, esto es, en el sentido de que ningún individuo pueda beneficiarse o recibir peor trato en función de sus creencias religiosas o su falta de ellas o como miembro de un grupo determinado que las profesa (Appiah 151-161). Pero la laicidad no tiene por qué implicar una actitud pública necesariamente pasiva. La cooperación del Estado con las distintas confesiones es, sin duda, admisible respecto de actividades de interés general en las que participan los grupos religiosos, tales como la educación, la sanidad o el patrimonio artístico. Pero, además, la laicidad debe 
suponer medidas positivas en favor de la libertad de los miembros de tales grupos que sean compatibles con el respeto de creencias y prácticas religiosas.

La libertad religiosa se corresponde con la vertiente trascendente de la libertad ideológica. Además de por el contenido de las ideas, la libertad religiosa se caracteriza por su ejercicio comunitario o colectivo (sin perjuicio de su componente individual) que alcanza su máxima expresión externa mediante los actos de culto. En este sentido, la libertad religiosa contiene un conflicto axiológico interno, entre el valor, consagrado por la tradición liberal de la idea de tolerancia, de la libertad de creencias del individuo como esencia de su autonomía, y el valor de las confesiones como entidades colectivas que permiten la búsqueda grupal de valores y creencias fundamentales y constituyen un ámbito de resistencia que desafía la autoridad moral del Estado (Appiah 142-144). El ejercicio de la libertad del individuo se produce en el seno de una entidad colectiva con autoridad sobre sus miembros.

Las concepciones institucionales o comunitaristas de lo religioso que asumen el segundo de los valores como central en la libertad religiosa tienden a favorecer el ocultamiento de la disidencia y la crítica individuales tras la reivindicación de la autoridad de la comunidad religiosa como tal en la definición del marco de valores y creencias. En este sentido de libertad religiosa, es función de un Estado laico respetar y favorecer las creencias de tales colectivos. Frente a la idea liberal del Estado como entidad situada en un ámbito distinto de las religiones y con la mera función de hacerlas convivir, las concepciones comunitaristas y pluralistas esperan del Estado el reconocimiento activo de la identidad religiosa. Se considera que, en la medida en que la religión es una de las formas sociales que confiere valor y sentido a la vida humana, su reconocimiento e inclusión contribuye a una visión más integradora de lo público. El Estado no trataría a todos los individuos con igual respeto si no hiciese lo posible por brindar igual respeto a las comunidades en las que se insertan y desarrollan. Desde estas concepciones, las restricciones externas a las comunidades religiosas a favor de los derechos de sus miembros aparecen como intromisiones ilegítimas en el modo en que cada confesión proyecta su modo de concebir y conocer el mundo.

Pero, en otro sentido, puede entenderse que el valor que está en la base de la libertad religiosa no es solo el contenido sustantivo que protege, la religión, ni el valor de la comunidad de creencias y doctrinas en último término, sino la autonomía individual «entendida como capacidad de buscar, comprometerse y desarrollar ideas, acciones y planes de vida... en referencia al ámbito de las creencias sobre el significado básico de la vida, la existencia o no de seres que nos trascienden, etcétera» (Ruiz Miguel 2013, 48). La laicidad tiene que 
integrar este segundo elemento de la libertad religiosa que prima la autonomía y la igualdad individuales. Y sobre esta base, la laicidad no implica una neutralidad entendida simplemente como inacción. Si un modelo de neutralidad pasiva supone que el Estado no debe comprometerse con ninguna creencia religiosa y debe actuar separado de las Iglesias, la garantía de la libertad religiosa puede requerir desde el punto de vista individual la intervención en el fomento del ejercicio libre y plural de las libertades de expresión, culto y creencias y, en general, de otros derechos que las comunidades religiosas pueden limitar u obstaculizar. La neutralidad como mera abstención no hace sino perpetuar situaciones de desigualdad y subordinación (Vázquez 2013, 11-15). Es esta interpretación la que permite eludir el riesgo de que la separación entre Estado e Iglesia se conciba de forma reductiva como independencia de la esfera religiosa respecto de las normas colectivas y el orden político.

\subsection{Laicidad y deliberación pública}

En segundo lugar, la laicidad puede entenderse como un complemento de la separación de poderes, en el sentido de que la religión se descarta como fuente de legitimación de las decisiones políticas, que deben tener su base en el principio democrático (Blancarte). La reivindicación de un Estado laico no se basa solo en la reclamación de mayor autonomía para desarrollar concepciones morales propias, sino también en la demanda de mayor capacidad para intervenir en la definición de las reglas colectivas como ciudadano en condiciones de igualdad con el resto y sin la imposición de doctrinas morales desde las confesiones religiosas mayoritarias. Esta concepción de la laicidad se enfrenta a la posición doctrinal de algunas Iglesias que consideran que la verdad moral que contienen está por encima de las leyes elaboradas colectivamente. Sus jerarquías intervienen directamente en la vida política tratando que las normas colectivas se conformen a la verdad moral que su Iglesia custodia (Bovero 14-15). Como afirma Luis Prieto, lo que sin duda excluye el Estado laico es la apelación al principio de autoridad para hacer valer posiciones públicas apelando simplemente a la doctrina religiosa, ética o filosófica particular de la que proceden (127-128).

La laicidad como modelo de convivencia social fundada sobre valores cívicos supone una perspectiva esencial para la integración igualitaria de las mujeres, en la medida en que la imposición del argumentario religioso en el discurso público ha servido históricamente para reforzar y legitimar la estructura patriarcal de la sociedad y la relegación de la mujer al ámbito privado. La autonomía de la política respecto de la religión supone que la regulación de aquellos aspectos privados y públicos de los que depende la superación de 
la subordinación de las mujeres debe desarrollarse en la deliberación pública mediante argumentos desligados de las doctrinas religiosas.

Los intentos de liberar el debate público de la presión de las Iglesias se vieron históricamente favorecidos por el proceso de secularización de las sociedades, esto es, la pérdida de relevancia de la religión en los distintos ámbitos de la vida social. Pero la realidad de nuestras sociedades es que solo relativa y transitoriamente han alcanzado un grado suficiente de secularización que sirva de fundamento a esa deliberación pública laica. El fenómeno religioso conforma en gran medida la moral social y sigue permeando las políticas de los Estados. En 2006 el Lobby Europeo de Mujeres manifestó su percepción de la creciente influencia de argumentos religiosos en la toma de decisiones por las instituciones públicas.

No encontramos en nuestra época los rasgos con los que Richard Bernstein caracterizó la secularización: diferenciación funcional, decadencia de la religión y privatización (Bernstein 170-171). Los debates de nuestros días sobre la «de-secularización» (Berger) y el «postsecularismo» (Habermas 2006, 2008) muestran una creciente complacencia con la influencia de las religiones en la formación de la opinión y la voluntad públicas, en la medida en que se piensa que las religiones portan contenidos y experiencias que son relevantes y fundan identidades y solidaridades que los individuos necesitan y valoran. Se considera que lo secular ha de ser sensible a la «fuerza de articulación» que tiene el lenguaje de la religión. El secularismo, afirma Mahmood (70), ofrece una comprensión empobrecida de las imágenes y signos religiosos, al no prestar atención a las prácticas y normas que las informan. Martha Nussbaum reconoce la necesidad de que seamos capaces de ver el mundo desde la perspectiva de la experiencia de los otros. La autora habla del cultivo sistemático de la «mirada mental», la imaginación comprensiva y empática que nos hace posible conocer cómo se ve el mundo desde el punto de vista de una persona de una religión o etnia diferente, evitando diseñar las políticas comunes desde una imagen generalizada del otro (2012, 175 y ss.). Sobre estas bases, conforme se amplíe lo discursivamente aceptable, la discusión política será capaz de integrar en mayor medida la complejidad de comprensiones sobre la vida humana. Tal ampliación favorecería la participación en la vida pública de los ciudadanos religiosos en tanto que tales y desde sus convicciones particulares, enriqueciendo el debate y favoreciendo la integración efectiva.

El postsecularismo responde a un contexto complejo en el que no solo resurge la religión en el ámbito público, sino que esa revalorización convive con su crítica en un contexto de pluralismo en el que un discurso público con vocación de universalidad se vuelve cada vez más problemático. En la 
medida en que el postsecularismo contribuye a disolver las dicotomías entre lo público y lo privado, la razón y los sentimientos, los derechos y las virtudes, evidenciando las contradicciones del pensamiento racionalista de la modernidad, sigue algunas de las líneas marcadas por la crítica feminista. Existe un feminismo que ha adoptado el giro postsecular introduciendo en su teoría una dimensión espiritual o una fe en la posibilidad de construir horizontes de normas y valores sociales alternativos mediante múltiples modos de interacción con los otros (Braidoti). Pero el riesgo de las posiciones que replantean la legitimidad de incorporar la religión al debate público es que tienden a interpretar la igualdad como reconocimiento de cada persona como miembro igual de una comunidad moral desde la que participa en el orden político (Beltrán 188). Es decir, se reconoce su autenticidad en la medida en que el individuo participa en una cultura o sistema compartido. El cambio en el sentido de la igualdad entraña serios riesgos para la continuidad de la legitimación de la subordinación de las mujeres, en la medida en que su participación puede verse mediada por posiciones religiosas o culturales que se anteponen a los argumentos sobre su libertad y derechos. Como afirma Elaine Graham, el género queda una vez más oculto en el debate sobre religión, política e identidad del postsecularismo (235).

No es función del Estado tutelar las «formas de vida», sean religiosas o no, sino los derechos de todos los ciudadanos. Y, en este sentido, como afirmó Paolo Flores D'Arcais, el Estado laico exige a todos realizar un esfuerzo de adaptación, eliminando de la deliberación pública «toda pretensión de cualquier "porque sí", expresiones de simple y totalitaria "voluntad de poder", incompatibles con la democracia» (58). Admitir una deliberación ampliada no puede hacerse sin establecer algunas restricciones relevantes. Como escribe Michelangelo Bovero, podríamos señalar dos límites a lo que sería admisible desde una cultura de la laicidad: en primer lugar, el antidogmatismo, es decir, la necesidad de promover un juicio crítico e independiente respecto de las afirmaciones o creencias avaladas por una autoridad. Y, en segundo lugar, un principio práctico de tolerancia, esto es, de ausencia de un deber de asumir determinadas creencias (16-17). La laicidad implica, pues, la apertura al convencimiento moral por medio de argumentaciones persuasivas sobre las normas a elegir como buenas. La exigencia de traducción de las reivindicaciones de los creyentes a un lenguaje laico no tiene por qué suponer una falta de respeto o reconocimiento, sino más bien, «una muestra de la idea de respeto mutuo, debido en la convivencia y necesario en la cooperación social, que en todo caso les permite seguir actuando de acuerdo con sus motivaciones religiosas» (Beltrán 180). 
En el ámbito político, ello implica el reconocimiento del disenso y la consideración del pluralismo como un hecho y como un valor. La regulación pública de aspectos que afectan especialmente a la libertad de las mujeres y tienen relevancia para las confesiones religiosas solo puede ser debatida en el foro público con una participación plural, sobre la base de argumentos que no apelen solo a verdades metafísicas y que puedan ser compartidas por todos. Y, en todo caso, permitiendo una amplia esfera de discrecionalidad individual que permita a cada uno realizar elecciones responsables (Bovero). La laicidad no nos posiciona para valorar aquello que es moralmente correcto o incorrecto sino aquello que está justificado que apliquemos coercitivamente de modo colectivo. Todas estas premisas están en la base de un feminismo como proyecto cívico, «visceralmente opuesto», como escribe Rosi Braidoti (3), al autoritarismo y la ortodoxia. Para éste la noción de agencia solo puede ser entendida en sentido crítico como productora de contra-subjetividades en un entorno de relaciones múltiples no mediadas por un único sistema de conceptos y significados.

El pensamiento laico, por tanto, no supone una ausencia de valores, sino que constituye una concepción ética que antepone la autonomía y las condiciones de legalidad a los diferentes credos y comunidades religiosas (Vazquez 2009, 100). Como afirma Ruiz Miguel, la laicidad apela a un discurso moral que conlleva criterios de corrección, tales como garantizar una deliberación colectiva racional, libre y pacífica (Ruiz Miguel 2007). La laicidad, por tanto, no asume necesariamente un relativismo moral, sino que aspira a la fundamentación racional del análisis crítico de las creencias o convicciones, favoreciendo su discusión racional y rechazando su imposición autoritaria o coactiva.

\subsection{Laicidad como protección de derechos básicos frente a la interferencia de lo religioso}

Por último, en tercer lugar, la laicidad no supone solo deberes que salvaguarden libertades esenciales de contenido ideológico, como la de expresión, culto o conciencia, sino que también debe entenderse como exigencia de garantizar, en general, otros derechos mediante la imposición de responsabilidades en la superación de las visiones discriminatorias que algunas confesiones han impuesto en las sociedades. Un Estado no laico no es solo el que trata de conformar las creencias de los ciudadanos, sino el que como consecuencia de su adscripción religiosa o de su actitud pasiva respecto de las tradiciones religiosas deniega o permite la vulneración de otros derechos a la ciudadanía. Como afirma Martha Nussbaum, la verdadera libertad religiosa requeriría que no se restringieran las otras libertades básicas que garantizan la dignidad de todo 
individuo $(1999,82)$. El conflicto ideológico del liberalismo con las dogmáticas religiosas no impidió que los Estados liberales aceptaran el rol que la religión desempeñaba en el mantenimiento del orden social a costa de la perpetuación de la estructura patriarcal que ello suponía. La separación Estado-Iglesia en lo público se interpretó compatible con la permanencia de la desigualdad en lo privado que las Iglesias seguían reforzando y legitimando.

Históricamente, el Estado laico ha sido compatible con ese papel fundamentador de la desigualdad que han desempeñado las religiones. En la medida en que muchas de las cuestiones esenciales para alcanzar la igualdad, que tradicionalmente se han considerado privadas, tienen relevancia pública, en un Estado laico deben estar enmarcadas en el contexto de normas colectivas que no estén determinadas en exclusiva por los dogmas de una Iglesia o una religión dominante. La laicidad es incompatible con la presión ilegítima que las Iglesias dominantes ejercen sobre la legislación al margen de los procedimientos formales de decisión pública y la colonización que pueden pretender sobre la moral social en materias especialmente sensibles para las mujeres. Es para ello para lo que el Estado laico debe proteger la libertad individual de conciencia y hacer posible la disidencia y la crítica interna y externa. Y debe, en concreto, proporcionar la legislación que haga posible la libertad de las mujeres para tomar decisiones propias en materia sexual, reproductiva, sanitaria, familiar, educativa, laboral, etc. sin estar coaccionadas por ideologías impuestas.

La religión condiciona en gran medida las vidas de las mujeres, creyentes o no, directamente o a través de su influencia sobre la moral social de las comunidades en las que se desarrollan. En muchas ocasiones esta influencia es desfavorable para los derechos y posición de las mujeres en cuanto contribuye a la construcción de estereotipos de género que refuerzan la subordinación de las mujeres en la sociedad. Al margen de las graves violaciones de derechos humanos que incitan y fundamentan las versiones más radicales de algunas doctrinas religiosas, estas pueden favorecer otras formas más sutiles de intolerancia y discriminación, contribuyendo a una cultura patriarcal que asuma el papel de la mujer como esposa, madre y ama de casa y obstaculice la adopción de reformas laborales y políticas ${ }^{1}$. Gran parte de la doctrina de las tradiciones religiosas gira en torno a las expectativas vinculadas a ser hombre o mujer, la comprensión y práctica de la sexualidad, o las condiciones de la vida en pareja y la procreación. Y en gran medida esas concepciones y prácticas tienen consecuencias negativas para la igualdad de las mujeres. Como afirma Susan Moller

1. Así lo reconoció la Resolución 1464 (2005) del Consejo de Europa sobre Mujeres y religión en Europa.

Feminismo/s 28, diciembre 2016, pp. 49-74 
Okin, gran parte de los rituales y prácticas de las grandes religiones están orientados a someter la sexualidad de las mujeres y su capacidad reproductiva al control de los hombres (668-669).

Las religiones son realidades fluidas cuyas prácticas e interpretaciones están arraigadas y varían en distintos contextos culturales e históricos. Las prácticas y normas que discriminan a las mujeres son en muchas ocasiones expresión de tradiciones y culturas asociadas a los dictados de una religión. Pero, entonces, ¿es realmente la religión o más bien la cultura la que contribuye a la endémica subordinación de las mujeres? La religión proporciona razones para fundar las prácticas y regulaciones que imponen a las mujeres conductas restrictivas de sus derechos. La religión es empleada, desde las diversas culturas, como justificación de imposiciones dirigidas solo a mujeres en relación con cuestiones relativas a la sexualidad y a la familia.

\section{RELIGIÓN, PODER Y MUJERES}

\subsection{Acerca de la pretendida peculiaridad de la identidad religiosa}

Las religiones no solo constituyen conjuntos de creencias en dogmas universales y sistemas institucionales sino que desempeñan uno de los más importantes modos de reivindicación identitaria. Las cuestiones relativas a las diferencias religiosas tienen en nuestras sociedades suficiente relevancia como para que la religión sea considerada, como la etnicidad o la nacionalidad, un elemento de las complejas identidades colectivas que nos sirven para juzgar el valor y sentido de nuestros planes de vida.

La concepción de que la religión es diferente a otras adscripciones de los individuos subyace a gran parte del debate sobre el papel de la religión en la vida pública. Su carácter especial implicaría la exigencia de mayor reconocimiento y protección públicas y su mayor peso en el balance de argumentos para la toma de decisiones públicas. Las razones que suelen invocarse al respecto son su valor de verdad, su contribución al bien común, y su aportación a la conformación de compromisos éticos últimos. Pero, como ha mostrado Amy Gutman, ninguna de estas razones habitualmente empleadas es suficiente para justificar el peso que los creyentes desean atribuir a la religión en la deliberación pública (Gutman, 2008: 218 y ss.). Respecto de la primera de las razones de su pretendida especialidad, la obvia imposibilidad de que todas las creencias religiosas puedan ser verdaderas al mismo tiempo implica la necesidad de excluir del debate democrático los argumentos con un fundamento exclusivamente religioso incapaces de ser traducidos en argumentos mutuamente justificables. La segunda de las razones apela al argumento común de que la 
identidad religiosa sirve un propósito público relevante en la medida en que ayuda a superar el individualismo egoísta favoreciendo la creación de un capital social y un sentido de reciprocidad. No obstante, las identidades religiosas no desempeñan necesariamente esta función, en la medida en que también pueden favorecer la intolerancia y la división, ni son condición suficiente para la promoción de un capital social, existiendo una pluralidad de factores sociales que favorecen igualmente el voluntariado y la solidaridad y que merecen, por tanto, igual promoción.

Por último, se aduce que las religiones no solo manifiestan una identidad sin más sino aquella que compromete en último término a una persona, integrando compromisos que se asumen como obligatorios por quienes creen en ellos (Song, 2008). Propiamente, lo que dota a esta argumentación de su fuerza no es que los compromisos éticos tengan su base en fundamentos teológicos cuanto el reconocimiento de los individuos como sujetos éticos cuyo sometimiento al orden político siempre es condicionado. Pero de esta premisa esencial lo que debe extraerse es que todas las convicciones éticas merecen igual respeto y consideración por las instituciones públicas. La democracia supone un modo legitimado de adoptar decisiones comunes en un contexto de profundos desacuerdos entre los compromisos éticos últimos de las personas. Aunque el respeto al sentido de lo moral de cada individuo es un presupuesto esencial de cualquier gobierno democrático, este no puede ser un valor absoluto en cuanto que puede entrar en conflicto con otros principios democráticos básicos. Como afirma Amy Gutman, «el acatamiento de la conciencia por sí mismo no ofrece garantía alguna de producir una mayor justicia en cualquier caso dado» $(2008,241)$.

Pero, además, el problema de la integración de lo religioso en la esfera pública es más complejo en la medida en que la articulación de los compromisos éticos que favorecen las religiones se produce dentro de un entramado institucional y cultural. El peso que se quiere atribuir a la religión en nuestros días no radica tanto en la intención de que se respete la conciencia individual cuanto en el reconocimiento de los grupos que la articulan y representan. Los argumentos que privilegian las identidades religiosas refuerzan el poder de la institución o grupo, debilitando la influencia de la esfera política como ámbito autónomo de decisión colectiva: los individuos participan como miembros del grupo que representa su interés fundamental. Aunque este riesgo de imposición autoritaria es común a cualquier grupo identitario, se refuerza en los grupos religiosos que se arrogan la representación de verdades morales o compromisos éticos últimos. Como afirma Martha Nussbaum, «no podemos aceptar la idea de que denegar cualquier derecho fundamental a cualquier individuo sea 
una legítima prerrogativa de ningún grupo religioso» (Nussbaum, 1999: 107). En este sentido, la Observación General n. ${ }^{\circ}$ 28, del Comité de los Derechos Humanos de Naciones Unidas, en su interpretación de la igualdad de derechos entre hombres y mujeres, afirmó que «no se puede invocar el artículo 18 para justificar la discriminación contra la mujer aduciendo la libertad de pensamiento, de conciencia y de religión».

\subsection{Las mujeres como minorías internas}

La dimensión colectiva de las prácticas religiosas, conforme a la que la identidad de cada uno se constituye de manera crucial a través de conceptos y prácticas que proporciona la religión, las acerca a las prácticas culturales generales. Si no existen razones suficientes para considerar que las religiones en esta dimensión colectiva constituyen un modelo especial de identidad es difícil trazar una línea divisoria entre prácticas religiosas y culturales. Con mayor razón si se considera que los significados de ambas cambian en las interacciones sociales; esto es, que también el sentido que se confiere a los compromisos éticos desde cada religión, como el sentido de las prácticas culturales, está abierto a procesos de resignificación y cambio en un contexto de contestación interna y externa mediante el que las voces disidentes plantean modos alternativos de interpretar los principios y prácticas (Benhabib 13).

Las prácticas religiosas se moldean conforme a las culturas en las que se desarrollan, del mismo modo que todas las culturas de algún modo se han creado y desarrollado conforme a concepciones religiosas. Como las culturas, las religiones ofrecen un contexto comunitario para la elección de modelos de acción. Si la función de las instituciones públicas no es decidir sobre la verdad moral o los compromisos éticos últimos de los individuos, su contribución a los grupos de creencias no ha de ser la asimilación ni la acomodación de las creencias cuanto el reconocimiento de la aportación de las religiones a la formación de estas y, por tanto, su promoción de la pluralidad y la no discriminación que permita la formación ética de todo individuo en libertad.

Conforme a estas premisas, el control de la no discriminación por parte de las instituciones públicas no se ha de limitar a garantizar la igualdad entre confesiones religiosas sino también la pluralidad y no discriminación internas. Una de las críticas comunes dirigidas a las tesis multiculturalistas plantea que la protección de los grupos y el reconocimiento de la obligación de no interferencia en sus asuntos internos puede reforzar la discriminación de sus miembros más vulnerables. Es lo que algunos autores han denominado el problema de las «minorías internas» o de las «minorías dentro de las minorías» (Green; Eisenberg y Spinner-Halev). La tolerancia liberal se tradujo de hecho 
en la existencia de áreas aisladas de la protección del poder político, en las que subsistían, sin embargo, estructuras de poder. Algunas de las normas y prácticas que han dominado esas áreas, como las familias o las religiones, se refieren a asuntos de género y sexualidad. De hecho, una de las formas usuales de defensa del propio grupo ante rápidos cambios sociales que lo amenazan es el reforzamiento de las restricciones a la libertad de las mujeres (Phillips 2005, 114).

La cuestión es compleja porque la necesidad de proteger «minorías internas» debe ponderarse con la necesidad de reconocer y proteger a la comunidad más amplia de la que forman parte. En muchos casos, esta comunidad constituye asimismo una minoría oprimida y la protección de las minorías internas puede emplearse para justificar la intervención externa del Estado sobre un grupo que él mismo oprime. Reconocer especiales poderes o inmunidades a las minorías internas puede suponer reforzar la posición de la comunidad más amplia respecto de las minorías (Green 263; Spinner-Haley). A diferencia de Jeff Spinner, sin embargo, no considero que la histórica discriminación a un grupo proporcione una justificación suficiente para la vulneración de los derechos de algunos de los miembros de ese grupo (Nussbaum 1999, 109). El problema reside en la legitimidad y la oportunidad de los modos de garantizar tales derechos sin reforzar con ello la posición de la comunidad mayoritaria que continua discriminando a la minoría y sin vulnerar el principio de neutralidad estatal.

Las dudas al respecto son difíciles de resolver: ¿quién y cómo debe intervenir?, ¿están los Estados liberal-democráticos suficientemente legitimados para intervenir en grupos con prácticas o normas iliberales?, ¿podemos confiar en que las autoridades estatales actuarán más positivamente a favor de los derechos de las minorías internas? Los grupos oprimidos -en cuyo seno puede haber minorías internas- suelen estar sub-representados en las comunidades más amplias de las que forman parte y resulta difícil justificar el asimilacionismo que supone que los órganos de decisión de éstas impongan sus códigos de conducta sobre sus miembros. La reforma externa de una norma de una comunidad minoritaria debe hacerse bajo ciertas condiciones: un clima general de respeto a la minoría sobre la que se va a intervenir, instrumentos de inclusión de las minorías en la deliberación pública de la sociedad más amplia que toma las decisiones a favor de los derechos de las minorías internas, atención a las voces de los afectados en estas, etc. En definitiva, las instituciones democráticas deben favorecer una esfera pública donde el reconocimiento de las diferencias y la contestación de las narrativas hegemónicas pueden tener lugar sin dominación (Benhabib 8). 
El Estado tiene una responsabilidad en la protección de los derechos de todos los individuos que no es completamente incompatible con el reconocimiento de cierto nivel de autonomía de las asociaciones intermedias. Ayelet Shachar considera que los modelos mixtos, como el de ciudadanía multicultural diferenciada que ella propone, permiten eludir la «paradoja de la acomodación multicultural» que muestra que los miembros oprimidos de algunos grupos, como las mujeres, están peor si el Estado reconoce y protege a las comunidades en las que se insertan (Shachar 2000, 2001). La libertad religiosa no es incompatible con la legítima función de las instituciones políticas, estatales e internacionales, de promover los elementos igualitarios de las tradiciones religiosas, incentivando la discusión interna y apoyando a los grupos internos que tratan de superar las discriminaciones. En el caso de las mujeres, esta función es complementaria del papel general que el Estado debe desempeñar, como ha escrito Cristina Sánchez, «en el reforzamiento de contextos que favorezcan la autonomía, la autoestima y la confianza de las mujeres» que son requisitos previos a cualquier opción fundada de pertenencia o salida de un grupo (Sánchez 300).

El carácter institucional de gran parte de las comunidades religiosas puede introducir elementos adicionales a la discusión sobre la intervención del Estado sobre las comunidades de creencias. Las confesiones religiosas no son solo sistemas de creencias y prácticas que operan informalmente, sino estructuras institucionales poderosas que tienen una especial capacidad para reforzar la discriminación sistémica de las mujeres. Como organizaciones que operan en el marco de sistemas político-jurídicos deberían ser objeto de control por parte de las instituciones públicas, tal como reconoce el art. 2e de la Convención sobre todas las formas de discriminación contra la mujer (en adelante, CEDAW en sus siglas en inglés). Especialmente cuando se reconocen a tales organizaciones exenciones a obligaciones generales o participación en subvenciones, éstas podrían condicionarse al cumplimiento de ciertas condiciones básicas de funcionamiento interno. Por ejemplo, en colegios concertados de confesión religiosa deben asegurarse de que la educación es conforme con los principios de igualdad de género, lo que no es siempre el caso. Es difícil determinar qué prácticas de instituciones religiosas se han de considerar discriminatorias a los efectos de condicionar las exenciones o subvenciones. ¿Se ha de considerar solamente el trato que dispensan en la prestación de servicios públicos o también el propio funcionamiento y organización interna de la institución religiosa? Esta cuestión debe plantearse en el debate público si se quiere reconocer seriamente la igualdad de género. En todo caso, resulta especialmente criticable que la diferente implantación institucional y arraigo social de las 
diferentes religiones influya en políticas estatales «incoherentes» que emplean criterios diferentes de tolerancia de la discriminación de género en función del apoyo social con que cuenta la confesión que la practica (Nussbaum 2012, 85, 127 y ss.).

\subsection{El conflicto género/religión como un problema de poder}

La religión, como otras dimensiones de las identidades colectivas, genera exigencias y expectativas que no todos pueden satisfacer de igual modo desde su situación y capacidades o que no sirven para atender de igual modo los intereses o necesidades de todos. En general, las religiones privilegian la posición de los hombres en su definición de lo bueno resultándoles a estos más adecuadas para conseguir sus objetivos. Ante este hecho, las mujeres se enfrentan al dilema de elegir entre su cultura o religión y sus derechos. La situación de verse obligado a abandonar la propia cultura o religión es arriesgada dado el valor de la pertenencia para los individuos. Appiah habla de «identificación» respecto del proceso mediante el que los individuos configuran su proyecto de vida y su concepto de una vida buena refiriéndose a categorías socialmente construidas. El individuo configura su vida pensando que una meta o una manera de actuar es apropiada para él como español, o mujer, o musulmán, o filósofo. Pensarse como E define su propia identidad. Y mediante su identidad el individuo hace que la historia de su vida se corresponda con patrones y narrativas más abarcadoras. Esta identidad social tiene relevancia ética (juega un papel en la definición, revisión y evaluación de los modelos que inspiran la vida propia) y política (desempeña un papel en el trato que los demás prodigan a los portadores de esa identidad) (Appiah 117, 119-121). El contexto identitario supone, pues, un marco necesario para la auto-realización y la capacidad de elección.

Pero, frente a este argumento relativo al valor de la cultura para el individuo, el problema surge precisamente cuando es la propia cultura la que menosprecia a ciertos sujetos y, entonces, las identidades colectivas se vuelven, no ya patrones en los que identificarse, sino límites para la realización de los propios planes de vida (Appiah, 175). Como afirma Okin, «al menos tan importante como el desarrollo del auto-respeto y la auto-estima por la propia cultura es el lugar que uno ocupa en el seno de esa cultura» (Okin 679-680). La necesidad de integración convierte en especialmente problemático el caso de quienes han desarrollado sus vidas entre los miembros de un grupo y se les niega, sin embargo, la pertenencia en igualdad al mismo (Margalit y Raz 444-445).

La respuesta liberal a la opresión en el seno de los grupos es la opción de salida. Si el grupo permite a los miembros abandonarlo en caso de considerarse 
discriminados o maltratados, se puede entender que quienes permanecen consienten indirectamente adherirse al grupo ${ }^{2}$. Pero, ¿es posible renunciar sin más a los grupos de identidad? La salida implica siempre una pérdida y un coste (Appiah 132-3). Es una opción tan arriesgada y dolorosa que no podemos suponer que quienes no abandonan el grupo permanecen por su propia voluntad, al menos no en un sentido que sirva como argumento suficiente para restringir los derechos que deberían corresponderles. El modo en que se configuran y funcionan grupos tan básicos como la familia, la Iglesia, el grupo étnico o la nación está precisamente orientado a retener a los miembros y obstaculizar su salida. Por ello, es un argumento insuficiente para estimar protegida la libertad de las mujeres. Por el contrario, tal libertad requiere el reconocimiento de restricciones y garantías que aseguren sus derechos en el seno de las comunidades de las que forman parte (Green 266). Las comunidades religiosas no suelen funcionar como asociaciones voluntarias respecto de las que se decide libremente entrar o salir como un club de tenis. Los miembros de estas confesiones en la mayoría de los casos han formado siempre parte de las mismas sin plantearse críticamente el sentido de su pertenencia y viéndose sometidos a una institución cuyo control y estructura está más allá de su alcance. En estas condiciones en que la entrada no es voluntaria y la salida implica un coste, las «minorías internas todavía necesitan sus derechos» (Green 267).

La opción de la salida pasa por alto que las comunidades de creencias, como en general las comunidades culturales, no son realidades estáticas y homogéneas sino conjuntos plurales de hábitos, significados, normas y valoraciones que tienen carácter dinámico. Evitar la salida de quienes tienen concepciones distintas de las hegemónicas supone instituir formas para que los disidentes e inconformistas puedan ejercer influencia dentro del propio grupo. Desde este punto de vista, la elección entre cultura y derechos no tiene por qué ser necesariamente una elección trágica. No lo es si se atiende al dinamismo inherente a la cultura y a los derechos, que pueden ser resignificados desde una pluralidad de posiciones. La cuestión se podría replantear en el sentido,

2. John Stuart Mill así lo entendió en su argumentación favorable a la tolerancia de la práctica mormónica de la poligamia, en la que afirma que es posible que esa minoría viva bajo las leyes propias con tal de que «concedan a los que no están satisfechos de sus procedimientos una perfecta libertad para separarse». Bien es cierto, sin embargo, que Mill considera que tales relaciones poligámicas son tan voluntarias para las mujeres como cualquier otra forma de institución matrimonial, en cuanto que las ideas y hábitos sociales, «enseñando a las mujeres a mirar el matrimonio como la única cosa necesaria, hacen concebible que muchas de ellas prefieran ser una de las varias esposas de un hombre a quedarse sin casar» (Mill 175). La salida se plantea como opción o posibilidad de elección para cualquiera que no esté satisfecho con las doctrinas o prácticas de la comunidad. 
como señala Shachar, de cómo pueden las mujeres modificar y renegociar su posición históricamente desaventajada mediante la producción y promoción de nuevos significados de los valores y normas del grupo que puedan llegar a ser hegemónicos (Shachar 2001, 65). La salida supone la necesidad de escapar de un entorno opresivo que es incapaz de reformarse desde dentro. Como señaló Young, que los individuos tengan que abandonar sus propias experiencias e identidad para adoptar un punto de vista general, ayuda a reforzar el privilegio, ya que el punto de vista y los intereses de los privilegiados tenderán a dominar el espacio público y a ocultar los de los otros grupos (Young 1989, 257).

Reconocer su carácter dinámico y heterogéneo debe hacernos rechazar la idea de la cultura como determinante absoluto de las elecciones y acciones individuales. La homogeneidad evidenciada por un grupo de identidad es el reflejo de una realidad encubierta de poder en la que no todos los individuos tienen igual capacidad para determinar qué valores son preponderantes y cómo se interpretan. Además, puede hablarse de otro aspecto que relativiza el valor de la cultura: el de la necesidad de plantearse si es posible definir algunos principios o criterios que limiten el tipo de acciones o decisiones que pueden evaluarse en función de los valores y normas de un grupo de identidad. Esto es, si existen límites a partir de los cuales resulta ilegítimo usar argumentos identitarios para defender una práctica o acción (Phillips 2007, 34). El liberalismo situaría ese límite en el daño; por ello, por ejemplo, se proscribe la ablación a mujeres (Ruiz Miguel 2007). Pero este límite es demasiado laxo. Los modos en que las religiones contribuyen a la discriminación por razón de género son muchas veces más sutiles y complejos. Como afirma Cass Sunstein, «algunas de las formas más perniciosas de discriminación sexual son resultado de prácticas de instituciones religiosas que pueden producir normas interiorizadas de subordinación» (4). Las mujeres no quieren solo un modelo de tolerancia de las religiones que garantice la evitación de daños sino ser reconocidas en condiciones de igualdad como sujetos con capacidad para deliberar acerca de cómo actuar y qué normas deben asumirse colectivamente. Y ello supone que pueden imponerse límites a las religiones no solo basadas en daños sino respecto de aquellas discriminaciones que condicionan su capacidad de acción y decisión.

El problema de la contradicción entre comunidades religiosas o culturales e igualdad de género no es principalmente un problema de contradicción en los principios o fundamentos sino un problema democrático de falta de participación en la configuración de las prácticas que interpretan esos principios. La legitimidad de las respuestas a los conflictos entre el género y la religión dependerá del grado en que contribuyan a ellas las voces de las mujeres. Como afirma Partha Chatterjee, si lo que se pretende lograr es un cambio progresivo 
en las prácticas sociales sancionadas por la religión, esa batalla debe comenzarse y ganarse en el seno de las propias comunidades religiosas; «no hay atajos históricos aquí», señala el autor (234). Las mujeres deben dejar de ser mero objeto de debate y pasar a ser agentes en el mismo, con un papel activo y reflexivo en su propia tradición religiosa. «Esta es exactamente», afirma Nussbaum, «la respuesta que una sociedad verdaderamente comprometida con la libertad religiosa debería alentar» $(1999,117)$. A las mujeres se les deniega la libertad religiosa si no se hace posible su capacidad de influir directamente en la estructura y contenido de la religión que elijan (Stuart, 2010).

El problema no es tanto un conflicto entre dos sistemas separados, el género y la religión, que deben ser mutuamente equilibrados, sino un problema de poder, esto es, de capacidad de imponer las propias interpretaciones de los valores y normas que condiciona al mismo tiempo cada uno de esos sistemas. Como afirma Phillips, no es la cultura -o la religión- la que determina un trato desigualitario a las mujeres, sino interpretaciones particulares de la tradición cultural, algunas veces invocadas deliberadamente para bloquear las pretensiones de igualdad de las mujeres $(2005,133)$. Y uno de los problemas más acuciantes, señala la autora, es que las demandas externas de igualdad de género están muchas veces entrelazadas con otros discursos que buscan fines de contenido desigualitario -anti-inmigración, anti-pueblos indígenas...- y se deslizan hacia discursos de superioridad cultural. Por ello, aquellas deben formularse desde la perspectiva política y contextual que evite que la reivindicación de la igualdad sea instrumentalizada (Agra).

La realidad es que muchas veces han sido las propias mujeres las que han utilizado los símbolos y tradiciones religiosas como instrumento para la inclusión en el espacio público y la incorporación al mismo de problemas que consideran relevantes. Seyla Benhabib ha mostrado, entre otros, el asunto del velo en las escuelas francesas como un ejemplo de empleo de símbolos propios de la esfera privada (culturales y religiosos) para desafiar las reglas de la esfera pública y así renegociar su significado y alcance (94-100). La identificación con la simbología y prácticas de una religión puede servir de instrumento para que las mujeres planteen en el debate público la necesidad de reinterpretar las normas y principios vigentes introduciendo valoraciones y perspectivas más complejas. Al reclamar respeto a sus creencias religiosas las mujeres tienen que dar justificaciones de sus acciones mediante razones asumibles en la esfera pública y clarificar en qué sentido interpretan sus principios y creencias y el modo en que éstas deben asumirse por la colectividad. De este modo, las mujeres se constituyen no solo en sujetos sino en agentes de lo político. 


\section{RELIGIÓN COMO EXCEPCIÓN A DERECHOS Y DISCRIMINACIÓN}

La interpretación más adecuada de la libertad religiosa supone, pues, asumir la legítima función de las instituciones públicas en la promoción del debate interno a las comunidades religiosas y la inclusión igualitaria. Esta interpretación supone asumir una concepción de la religión no principalmente como comunidad de creencias sino como comunidad de conversación, en la que se contribuye colectivamente a la formación de compromisos éticos. Así concebida, la aportación de las instituciones públicas no se debe orientar sin más a la preservación y acomodación de creencias dadas cuanto a la promoción de la libertad efectiva para asumir compromisos vinculantes. Lo que supone la necesidad de favorecer la igualdad en la elección de las creencias y en la inclusión en las comunidades que las conforman. Quiero concluir este trabajo mostrando cómo algunos de los instrumentos que se emplean para proteger las creencias religiosas asumen una concepción estática y homogénea de las religiones, que desatiende y refuerza el modo en que estas afectan a la igualdad de las mujeres y ocultan el debate interno que condiciona el peso de los argumentos religiosos.

Entre tales argumentos se puede mencionar, en primer lugar, las excepciones religiosas a la aplicación de leyes generales. En la línea de la tradición americana de la acomodación, se piensa que, puesto que las leyes suelen encarnar las ideas de quienes representan la voluntad mayoritaria, pueden resultar incompatibles con la comprensión del mundo y las creencias de las minorías. La protección de miembros de estas minorías requiere no solo su reconocimiento en condiciones de igualdad a sostener libremente sus creencias sino también la necesidad de exenciones especiales en función de su carácter minoritario. De este modo, se trata de que el Derecho se acomode a las creencias de los individuos en la máxima medida compatible con el orden y los intereses generales. Este mecanismo de acomodación prioriza la conciencia individual sobre la voluntad legislativa y se suele realizar mediante la ponderación judicial de los principios relativos a la religión y la conciencia con los principios que protegen el orden e intereses generales.

El problema es que ese reconocimiento de exenciones puede suponer la desobediencia a leyes generales que protegen especialmente a las mujeres pero resultan inasumibles para algunas confesiones religiosas. Ciertamente, el modelo de exenciones individualizadas permite atender formas diversas de discriminación que obedecen a factores plurales y particularizados, como reclaman los estudios recientes sobre discriminación múltiple e interseccional. Pero, a pesar de la ventaja que esa perspectiva supone para comprender en su complejidad la realidad de la discriminación, puede acabar diluyendo la 
categoría de género y su capacidad de denunciar un modelo de opresión que permea todas las sociedades. Además, los argumentos religiosos tendrían que estar en pie de igualdad con otras razones que las personas puedan tener para ser objeto de exenciones. Y, en concreto, la religión no es diferente de otras circunstancias que constituyen la base de discriminaciones complejas que alimentan la subordinación sistémica de las mujeres. Atender solo a exenciones por motivos religiosos supone tratar desigualitariamente a aquellos individuos que tienen razones diferentes e igualmente fundadas para solicitar que el sistema se acomode a sus necesidades.

En segundo lugar, también la religión ha servido de base para argumentar a favor de la denominada «defensa cultural» en Derecho penal, que constituye otro instrumento cuya pretensión de garantía de las identidades colectivas supone la desprotección de las mujeres. La defensa cultural ha sido muy debatida en la cultura jurídica anglosajona y en los debates feministas sobre multiculturalismo. Es un concepto que no se emplea en el ámbito del Derecho europeo continental. Podría decirse que en este ámbito no se corresponde con una figura penal en particular, sino la suma de opciones diversas, como causa de justificación, inimputabilidad, error de prohibición o causa de atenuación en cuyo uso pueden emplearse argumentos culturales o religiosos. En el ámbito anglosajón ha adoptado formas muy diversas de argumentación, como las relativas al peso del contexto cultural y los estereotipos de género en la asignación de significado a las acciones de las mujeres, o la consideración como argumento legítimo de defensa de aquel que tiene en cuenta una distinta percepción del delito por el agente como consecuencia de su identidad cultural (Phillips 2003). La especial trascendencia y relevancia de las creencias religiosas se invocan muchas veces en este tipo de defensa para interpretar los hechos de un caso y justificar acciones que de otro modo constituirían delitos.

Como ha destacado la crítica feminista, estos argumentos de defensa conducen a un trato más mitigado de los delitos contra las mujeres y a la infra-valoración de las mujeres de las culturas minoritarias, que quedan invisibilizadas en las tradiciones religiosas asumidas como coherentes e incontestadas. Pero el problema, como afirma Anne Phillips, va más allá, en cuanto que la defensa cultural es eficaz cuando se produce en el marco de una estructura discriminatoria más amplia. Es solo en aquellos contextos en que la cultura y moral mayoritarias asumen concepciones sexistas de las ideas de agencia y responsabilidad, en los que resulta eficaz el argumento de la defensa cultural. Lo que es consecuencia de que el género no es sino una construcción social en todo caso, que también en las culturas mayoritarias sigue definiendo de modo subordinado lo femenino frente a lo masculino. En este sentido, resulta 
paradójico que el rechazo a la defensa cultural se produce en un contexto general de prejuicios discriminatorios en relación con el género. «Los usos y abusos de la defensa cultural destacan, por tanto, cuestiones que tienen una procedencia mucho más amplia y deberían orientarnos a desafiar de modo completo las normas patriarcales allí donde estén presentes» (Phillips 2003, 531).

Por último, las reservas a los documentos jurídicos internacionales de protección de las mujeres también relativizan su eficacia en aras a la protección del contexto cultural y religioso de cada Estado. La interpretación que se ha hecho hasta aquí de la libertad religiosa en el sentido de incluir el derecho de las mujeres a participar en la estructura y contenido de la misma es la única que resulta conforme con la obligación jurídica de los Estados de garantizar conjuntamente la igualdad de género y la libertad religiosa de las mujeres. Las obligaciones de los Estados respecto del reconocimiento y protección de las creencias y prácticas religiosas quedan condicionadas por su compromiso, expresado en el art. 2.f) de la CEDAW, de «adoptar todos las medidas adecuadas, incluso de carácter legislativo, para modificar o derogar leyes, reglamentos, usos y prácticas que constituyan discriminación contra la mujer» y, conforme al artículo 5, «modificar todos los patrones socioculturales» discriminatorios.

La Convención es uno de los documentos internacionales que ha recibido mayor número de reservas con vocación de modificar o excluir los elementos básicos del tratado. Las reservas pretenden facilitar la incorporación a los tratados del mayor número posible de Estados, adaptando sus circunstancias particulares a los objetivos de la norma. Las reservas a la Convención se han justificado sobre la base de que una actitud intolerante de exclusión de los países que no satisfacen los estándares específicos de la Convención puede ser ineficaz y contraproducente para el avance de los derechos de las mujeres, debiéndose en cambio tratar de lograr un desarrollo progresivo de aproximación a las exigencias del tratado en función de las circunstancias culturales, religiosas, políticas o económicas de cada Estado (Cook 1990, 679 y ss). En cambio, si la reserva evidencia, en contra de los dispuesto en el artículo 28.2 de la CEDAW, un propósito genérico de no sentirse vinculado por el objeto básico de la Convención resulta inaceptable y debería rechazarse.

La gran mayoría de reservas a la Convención han tenido este carácter general, ilimitado e indefinido, y han condicionado la interpretación de sus disposiciones a su compatibilidad con los preceptos de la religión dominante en el Estado que la formula. Este tipo de reservas socava las bases del Derecho internacional convencional. Es la aceptación amplia de la Convención la que le proporciona la credibilidad creciente que hará posible que se constituya en norma consuetudinaria, capaz de convertirse en un deber prima facie respecto 
de cualquier Estado incluso no signatario en la medida en que su generalización aumenta la legitimidad del orden internacional.

El objeto último de los documentos que conforman el Derecho internacional de los derechos humanos no es que cada Estado trate de conformarse a los mismos desde sus circunstancias particulares, sino favorecer un marco normativo progresivamente ampliado que vincule al mayor número de instituciones en la protección de los derechos básicos de todas las personas. Las reservas a la CEDAW asumen que su generalidad está más condicionada que otros tratados de derechos humanos porque su objeto es culturalmente más sensible. Encontramos aquí de nuevo un contexto general dominado por la consideración de la igualdad de género como secundaria respecto de intereses e identidades colectivas y la instrumentalización de los derechos de las mujeres en los conflictos interculturales a favor de discursos de contenido desigualitario. De este modo, la Convención, que nació con la vocación de ser el principal documento de lucha contra la discriminación de las mujeres, reconoce la discriminación como un asunto jurídico, pero condiciona su superación a un desarrollo progresivo que queda en manos de la buena voluntad y el cambio de actitudes. La tolerancia a las reservas no hace sino restringir más el ya limitado alcance de la Convención (Charleworth, Chinkin y Wright, 634).

\section{REFERENCIAS BIBLIOGRÁFICAS}

Agra, María Xosé. «Multiculturalidad, género y justicia». Miradas multidisplinares para un mundo en igualdad. Ponencias de la I Reunión Científica sobre Igualdad y Género. Coord. Clavo, M. ${ }^{a}$ Josefina y M.. Angeles Goicoechea. Logroño: Servicio de Publicaciones de la Universidad de la Rioja, 2014, 77-98.

Appiah, Kwame Anthony. La ética de la identidad. Buenos Aires: Katz, 2007.

Beltrán, Elena «Ciudadanía reticente y el significado de respeto». Dilemata 10 (2012):173-192.

Benhabib, Seyla. The Claims of Culture. Equality and Diversity in the Global Era. Princeton: Princeton University Press, 2002.

Berger, Peter L. (1999). «The desecularization of the world: a global overview». The desecularization of the world. Resurgent religion and world politics. Ed. Peter L. Berger. Washington: Ethics and Public Policy Center, 1999, 1-18.

Bernstein, Richard J. El abuso del mal. La corrupción de la política y la religión desde el 11/9. Buenos Aires: Katz, 2006.

Blancarte, Roberto. «Género, mujeres y Estado laico». Género, Derecho y Justicia. México: Suprema corte de Justicia de la Nación, 2011.

Bovero, Michelangelo. El concepto de laicidad. México: UNMA-Cátedra Benito Juárez-Instituto de Investigaciones Jurídicas-Instituto Iberoamericano de Derecho Constitucional, 2013. 
Braidotti, Rosi. «In Spite of the Times: The Postsecular Turn in Feminism». Theory, Culture E Society, 25.6(2008): 1-24.

Charlesworth, Hilary, Christine Chinkin y Shelley Wright. «Feminist Approaches to International Law». The American Journal of International Law, 85.4 (1991): 613-645.

Chatterjee, Partha. «Secularism and Toleration». Empire and Nation. Selected Essays. Nueva York: Columbia University Press, 2010, 203-235.

Cook, Rebecca J. «Reservations to the Convention on the Elimination of All Forms of Discrimination Against Women». Virginia Journal of International Law, 30 (1990): 643-716.

Eisenberg, Avigail y Jeff Spinner-Halev (eds.). Minorities within Minorities: Equality, Rights, and Diversity. Cambridge: Cambridge University Press, 2005.

European Women's Lobby. «Religions and Women's Human Rights». Position Paper of the European Women's Lobby. Adoptado el 27 de mayo de 2006.

Flores D'Arcais, Paolo. «Once tesis contra Habermas». Claves de Razón Práctica, 179 (2008): 56-60.

Graham, Elaine. «What's Missing? Gender, Reason and the Post-Secular». Political theology, 13.2 (2012): 233-245.

Green, Leslie. «Internal Minorities and their Rights». En Kymlicka, W., The rights of minority cultures. Ed. Kymlicka, Will. Oxford: Oxford University Press, 1995

Gutman, Amy. La identidad en democracia. Madrid: Katz, 2008.

Habermas, Jürgen. Entre naturalismo y religión. Barcelona: Paidós, 2006.

Habermas, Jürgen. «La voz pública de la religión». Claves de razón práctica, 180, (2008): 4-6

Mahmood, Saba. «Religious reason and the secular affect: An incommensurable divide?» Is critique secular? Blasphemy, injury, and free speech? Eds. Asad, Talal, Wendy Brown, Judith Butler, y Saba Mahmood. Berkeley: University of California, 2009, 64-100.

Margalit, Avishai y Raz, Joseph (1990). «National Self-Determination». The Journal of Philosophy, 87.9 (1990): 439-461.

Mill, John Stuart. Sobre la libertad. Madrid: Alianza, 1970.

Nussbaum, Martha. Sex and Social Justice. Oxford: Oxford University Press, 1999.

Nussbaum, Martha. La nueva intolerancia religiosa. Cómo superar la política del miedo en una época de inseguridad. Barcelona: Paidós, 2012.

Okin, Susan M. «Feminism and Multiculturalism: Some Tensions». Ethics, 108 (1998): 661-84.

Phillips, Anne. «When Culture Means Gender: Issues of Cultural Defence in the English Courts». The Modern Law Review, 66.4 (2003): 510-531.

Phillips, Anne. «Dilemmas of Gender and Culture: the Judge, the Democrat, and the Political Activist». Minorities within Minorities: Equality, Rights, and

Feminismo/s 28, diciembre 2016, pp. 49-74 
Diversity. Eds. Eisenberg, Avigail y Jeff Spinner-Halev. Cambridge: Cambridge University Press, 2005.

Phillips, Anne. Multiculturalism without Culture. Princeton: Princeton University Press, 2007.

Prieto, Luis. «Religión y política (A propósito del Estado laico)». Persona y Derecho, 53 (2005): 113-138.

Ruiz Miguel. Alfonso. «Laicidad, laicismo, relativismo y democracia». Sistema: Revista de ciencias sociales, 199 (2007): 39-60.

Ruiz Miguel, Alfonso. Laicidad y constitución. México: Universidad Nacional Autónoma de México, 2013.

Sánchez Muñoz, Cristina. «Negociaciones culturales y género: hacia un feminismo transnacional». Entre Estado y Cosmópolis. Derecho y justicia en un mundo global. Ed. Ruiz Miguel, Alfonso. Madrid: Trotta, 2014, 289-313.

Shachar, Ayelet. «Should Church and State Be Joined at the Altar? Women's Rights and the Multicultural Dilemma». Citizenship in Diverse Societies. Eds. Kymlicka, Will y Wayne Norman. Oxford: Oxford University Press, 2000, 199-223.

Shachar, Ayelet. Multicultural Jurisdictions: Cultural Differences and Women's Rights. Cambridge: Cambridge University Press, 2001.

Song, Sarah. "The Subject of Multiculturalism: Culture, Religion, Language, Ethnicity, Nationality, and Race?». New Waves in Political Philosophy. Eds. Bruin, Boudewijn y Christopher F. Zurn. Nueva York: Palgrave MacMillan, 2008, 177-197.

Spinner-Halev, J. «Feminism, Multiculturalism, Oppression, and the State». Ethics, 112.1 (2001): 84-113.

Stuart, Alison. «Freedom of Religion and Gender Equality: Inclusive or Exclusive». Human Rights Law Review 10:3 (2010): 429-459.

Sunstein, Cass R. «On the Tension between Sex Equality and Religious Freedom». Public Law and Legal Theory Working Papers. University of Chicago Law School, 167 (2007): 1-20.

Vázquez Rodolfo. «Laicidad, religión y deliberación pública». Las fronteras morales del derecho. México: Fontamara, 2009, 93-111.

Vázquez, Rodolfo. Democracia y laicidad activa. México: UNMA-Cátedra Benito Juárez-Instituto de Investigaciones Jurídicas-Instituto Iberoamericano de Derecho Constitucional, 2013.

Young, Iris Marion. «Polity and Group Difference: A Critique of the Ideal of Universal Citizenship». Ethics, 99.2 (1989): 250-274.

Young, Iris Marion. «Structural Injustice and Politics of Difference». Intersectionality and Beyond. Law, Power and the Politics of Location. Eds. Grabham, Emily, Davina Cooper, Jane Krishnadas y Didi Herman. Abingdon: RoutledgeCavendish, 2009, 273-298. 